\title{
Insider trading with partially informed traders
}

\author{
Knut K. Aase, Terje Bjuland and Bernt Øksendal \\ *Knut.Aase@NHH.NO, Terje.Bjuland@nhh.no, oksendal@math.uio.no
}

November 15, 2011

\begin{abstract}
The single auction equilibrium of Kyle's (1985) is studied, in which noise traders may be partially informed, or alternatively they can be manipulated. Unlike Kyle's assumption that the quantity traded by the noise traders is independent of the asset value, we assume that the noise traders are able to correlate their trade with the true price. This has several implications for the equilibrium, one being that the insider's expected profits decrease as the noise traders' ability to correlate positively improve. In the limit, the noise traders do not lose on average, and the insider makes zero expected profits. When the correlation is negative, we interpret this as manipulation. In this case the insider makes the highest expected profits, and the informativeness of prices is at its minimum.
\end{abstract}

KEYWORDS: Insider trading, asymmetric information, strategic trade, correlated trade, partially informed noise traders

\section{Introduction}

In his seminal paper on insider trading, Albert Kyle (1985) asks several questions: How valuable is private information to an insider? How does

*Norwegian School of Economics 5045 Bergen, Norway and Centre of Mathematics for Applications (CMA), University of Oslo, Norway. The research leading to these results has received funding from the European Research Council under the European Community's Seventh Framework Programme (FP7/2007-2013) / ERC grant agreement no [228087] 
noise trading affect the volatility of prices? What determines the liquidity of a speculative market? He provides answers to these and other questions by modeling rigorously the trading strategy of an insider in a model of efficient price information.

We focus on the single auction model in which one risky asset is exchanged for a riskless asset among three kinds of traders: a single insider who has access to private observation of the ex post liquidation value of the risky asset: less informed noise traders who must trade; and market makers who set prices conditional on information they have about the quantities traded by others. Trading is modeled as a single auction.

In the standard model the noise traders can be considered as less than fully rational, since the average profits of the insider is exactly what the noise traders lose, while the market makers are making zero profits. The market makers cannot distinguish the trading of the insider from the trading of the noise traders, who in effect provide camouflage which enables the insider to make profits on their expense.

In economics one ideally prefers all agents in the model to be rational, like in game theory, but when modeling insider trading this goal is difficult to fulfill. The rational expectations equilibrium models assume that traders maximize expected utility with rational beliefs, where the latter are defined to be consistent with the model itself. Noise was not viewed as stemming from incorrect beliefs, was somewhat vague and corresponded to a random error term added to the aggregated excess demand function. The existence of noise traders was intitially put forth as a solution to the "no trade" result of Grossman and Stiglitz (1980). Understanding the role of "noise" appeared to require a different framework, and was done by Kyle (1985) and Glosten and Milgrom (1985). These authors identified certain people as trading in a way which makes noise in a sense that their trade is not based on information.

A reasonable response to the presence of insiders in a market would be to avoid trading in this market altogether. In real stock markets people may have an idea that insiders exist, yet trade takes place in these markets. Liquidity (noise) traders presumably trade for other reasons than maximizing profits, one being that they simply need liquidity. In general the situation may be viewed to have a certain resemblance to gambling. Although people know that on average they lose, still some lucky ones do win, which motivates some people to participate.

The details of the identity of noise traders or liquidity traders were initially left vague. Notably, noise traders were modeled as equally likely to be 
buying or selling securities, which while making models technically tractable, is counterintuitive. Exogenous reasons for needing money, and hence having to sell securities seem more natural than exogenous reasons for having to buy securities.

Both the standard model and the model we suggest can be seen as an attempt to model reality as rigorously as possible, subject to certain limitations. Our framework is, however, closer to the ideal of game theory than the standard one, since we allow the noise traders to be partially informed.

The degree of information held by the noise traders is measured by a correlation coefficient, and when this takes its maximum value of one, they are in effect equally informed as the insider, in which case all participants in the market make zero profits on average. Thus the noise traders in our model are not equally likely to be selling or buying securities.

In the present model the noise traders may also correlate their trade negatively with the true value. The reason for this is outside the model, but if this happens, the insider makes more profits than in the uncorrelated case at the noise traders expense. Negative correlation can occur for different reasons, one being that the insider, who has privileged information, is able to mislead the noise traders.

Such situations may seem unlikely to fit into a neo-classical equilibrium, but as we demonstrate, it is sustainable in the framework of insider trading.

Originally we attempted to describe these phenomena in a continuous time context as in Aase et. al. (2011b), which would allowed us to study other questions as well, related to a dynamic economy, like how does colored noise trading affect the volatility of prices? Also smart money would then have a limited horizon over which trade can occur. This model is related to the one in Back (1992), as well as the continuous version in Kyle (1985), but is a more direct approach. However, confronted by some technical difficulties in connection with the associated filtering theory (e.g., Aase et. al. (2011a)), we decided to first study a single auction.

There is a vast literature on insider trading, and in particular the question of who the noise traders are. It is the hope that our paper will shed some further light on this latter question. Regarding the topic of rationality, Spiegel and Subrahmanyam (1992) replaced Kyle's uninformed liquidity traders with strategic utility-maximizing agents trading for hedging purposes. Diamond and Verrecchia (1981) suggest adding a noise term to agents' risk exposures. Risk-averse agents will then have an insurance motive for trading. De Marzo and Duffie (1999) propose a model where different traders have different dis- 
count rates. These papers solve the problem of finding a logically consistent model that can be used for e.g., welfare statements, of markets with imperfect information revelation.

DeLong, Shleifer, Summers and Waldman (1990), Dow and Gorton (1994), and Shleifer and Vishny (1997) propose limits to arbitrage in order to explain that noise traders are not eliminated by informed traders. The view that noise traders are less than rational is discussed in Shiller (1984), Schleifer and Summers (1990) and Barberis and Thaler (2003). Admati and Pfleiderer (1988) introduce two types of liquidity traders, discretionary and nondiscretionary. Dow and Gorton (2006) present a broad review of various aspects of noise traders, and conclude that the their identities, motivations and ability to persist remain topics of research.

The paper is organized as follows: In Section 2 we find an equilibrium in a one period model with correlated noise trades, in Section 3 we discuss the properties of the equilibrium, and Section 4 concludes.

\section{A single auction equilibrium}

In this section we find an equilibrium in the one period model with correlated trades. The structure of the model is the following: There are two dates, 0 and $T$. The risky asset value $\tilde{v}$ is normally distributed at date 0 with mean $p_{0}$ and variance $\sigma_{v}^{2}$, in short, $\tilde{v} \sim \mathcal{N}\left(p_{0}, \sigma_{v}^{2}\right)$. The insider knows the date $T$ realization $\tilde{v}^{\text {obs }}=v$ at time 0 , the others observe $\tilde{v}$ at $T$ to be $v$. The quantity traded by the noise traders is $\tilde{z}$, where $\tilde{z} \sim \mathcal{N}\left(0, \sigma_{z}^{2}\right)$. While Kyle (1985) assumes $\tilde{z}$ and $\tilde{v}$ to be independent, we assume $(\tilde{z}, \tilde{v})$ to be jointly normally distributed with correlation coefficient $\rho$. One interpretation is that when $\rho>0$, the noise traders are more rational than in the standard model. If $\rho<0$ on the other hand, we interpret this as manipulation, i.e., the noise traders have been manipulated by the other market participants. The quantity traded by the insider is $\tilde{x}$, the price is denoted $\tilde{p}$.

Trading is structured in two steps as follows: In step one, the exogenous values of $\tilde{v}$ and $\tilde{z}$ are realized and the insider chooses the quantity $\tilde{x}$ he trades. In doing so, he observes $\tilde{v}^{o b s}=v$, but not $\tilde{z}$. The insider's trading strategy is given by some real, measurable function $x: R \rightarrow R$, i.e., $\tilde{x}=x(\tilde{v})$.

In step two the market makers determine the price $\tilde{p}$ at which they trade the quantity necessary to clear the market. In doing so they observe $(\tilde{x}+$ $\tilde{z})$ but not $\tilde{x}$ or $\tilde{z}$ separately. Their pricing rule is determined by a real, 
measurable function $p: R \rightarrow R$ so that $\tilde{p}=p(\tilde{x}+\tilde{z})$. The insider's profit is denoted by $\tilde{\pi}$, where $\tilde{\pi}=(\tilde{v}-\tilde{p}) \tilde{x}$. Since $\tilde{\pi}$ and $\tilde{p}$ depend upon the real functions $x(\cdot)$ and $p(\cdot)$, we may write $\tilde{\pi}=\tilde{\pi}(x, p)$ and $\tilde{p}=\tilde{p}(x, p)$. We assume risk neutrality.

Equilibrium. An equilibrium is a pair of functions $(x, p)$ such that the following two conditions hold:

(1) Profit maximization: For any other trading strategy $x^{\prime}$ and for any $v$

$$
E\{\tilde{\pi}(x, p) \mid \tilde{v}=v\} \geq E\left\{\tilde{\pi}\left(x^{\prime}, p\right) \mid \tilde{v}=v\right\}
$$

(2) Market efficiency: The market price $\tilde{p}$ satisfies

$$
\tilde{p}(x, p)=E\{\tilde{v} \mid \tilde{x}+\tilde{z}\} .
$$

This model is not purely game theoretic, because the noise traders do not explicitly maximize any particular objective. However, in our model they may act rational, as we shall see. Our main result is the following:

Theorem 2.1. There exists a unique equilibrium in which $x$ and $p$ are linear functions. Defining

$$
\beta=\frac{1}{2} \frac{\sigma_{z}}{\sigma_{v}}\left(\sqrt{4-3 \rho^{2}}-\rho\right) \quad \text { and } \quad \lambda=\frac{\sigma_{v}}{\sigma_{z} \sqrt{4-3 \rho^{2}}}
$$

the equilibrium is given by

$$
x(\tilde{v})=\beta\left(\tilde{v}-p_{0}\right), \quad p(\tilde{x}+\tilde{z})=p_{0}+\lambda(\tilde{x}+\tilde{z}) .
$$

Proof. We conjecture a liner equilibrium of the following type:

$$
p(y)=\mu+\lambda y, \quad(y=x+z), \quad x(v)=\alpha+\beta v
$$

for some constants $\mu, \lambda, \alpha$ and $\beta$. Given the linear rule $p(\cdot)$, the insider's profits can be written

$$
E\{[\tilde{v}-p(\tilde{x}+\tilde{z})] x \mid \tilde{v}=v\}=\left[v-\mu-\lambda x-\lambda \rho \frac{\sigma_{z}}{\sigma_{v}}\left(v-p_{0}\right)\right] x,
$$

which follows, since by our assumption that $(\tilde{z}, \tilde{v})$ is binormal,

$$
E\{\tilde{z} \mid \tilde{v}=v\}=\rho \frac{\sigma_{z}}{\sigma_{v}}\left(v-p_{0}\right)
$$


by the projection theorem. Profit maximization of this quadratic objective requires that $x$ solves

$$
v-\mu-\lambda \rho \frac{\sigma_{z}}{\sigma_{v}}\left(v-p_{0}\right)-2 \lambda x=0
$$

or

$$
x=\left(\frac{1}{2 \lambda}-\frac{1}{2} \rho \frac{\sigma_{z}}{\sigma_{v}}\right) v+\frac{1}{2} \rho \frac{\sigma_{z}}{\sigma_{v}} p_{0}-\frac{1}{2 \lambda} \mu .
$$

In other words, $x(v)=\beta v+\alpha$ where the constants $\alpha$ and $\beta$ are given by

$$
\alpha=\frac{1}{2} \rho \frac{\sigma_{z}}{\sigma_{v}} p_{0}-\frac{1}{2 \lambda} \mu \quad \text { and } \quad \beta=\frac{1}{2 \lambda}-\frac{1}{2} \rho \frac{\sigma_{z}}{\sigma_{v}} .
$$

Given $x$ and $p$, the market efficiency condition is equivalent to $(\tilde{y}=\tilde{x}+\tilde{z})$

$$
\tilde{p}(x, p)=\mu+\lambda y=E\{\tilde{v} \mid \alpha+\beta \tilde{v}+\tilde{z}=y\} .
$$

Proceeding as above, this expression is computed as follows:

$$
\tilde{p}(x, p)=p_{0}+\rho_{X, Y} \sigma_{v} \frac{y-\left(\alpha+\beta p_{0}\right)}{\sqrt{\beta^{2} \sigma_{v}^{2}+\sigma_{z}^{2}+2 \beta \rho \sigma_{z} \sigma_{v}}},
$$

where

$$
\rho_{X, Y}=\frac{\operatorname{cov}(\tilde{v}, \alpha+\beta \tilde{v}+\tilde{z})}{\sigma_{v} \sqrt{\beta^{2} \sigma_{v}^{2}+\sigma_{z}^{2}+2 \beta \rho \sigma_{z} \sigma_{v}}} \text { and } \operatorname{cov}(\tilde{v}, \alpha+\beta \tilde{v}+\tilde{z})=\beta \sigma_{v}^{2}+\rho \sigma_{z} \sigma_{v} .
$$

Hence the pricing rule is filtered out as

$$
\tilde{p}(x, p)=p_{0}+\frac{\beta \sigma_{v}^{2}+\rho \sigma_{z} \sigma_{v}}{\beta^{2} \sigma_{v}^{2}+\sigma_{z}^{2}+2 \beta \rho \sigma_{z} \sigma_{v}}\left(y-\left(\alpha+\beta p_{0}\right)\right) .
$$

Comparing to (2.2) the constants $\mu$ and $\lambda$ are given by

$$
\mu=p_{0}-\lambda\left(\alpha+\beta p_{0}\right) \quad \text { and } \quad \lambda=\frac{\beta \sigma_{v}^{2}+\rho \sigma_{z} \sigma_{v}}{\beta^{2} \sigma_{v}^{2}+\sigma_{z}^{2}+2 \beta \rho \sigma_{z} \sigma_{v}} .
$$

In order to find the equilibrium, we have to solve the four equations given in (2.1) and (2.3) in the four unknowns $\alpha, \beta, \mu$ and $\lambda$.

Starting with (2.1) we notice that $\alpha+\beta p_{0}=\left(p_{0}-\mu\right) / 2 \lambda$, and inserted in the first of the equations in $(2.3)$ we get $\mu-p_{0}=\left(\mu-p_{0}\right) / 2$ which implies that $\mu=p_{0}$. The first of the equations in (2.1) can therefore be written

$$
\alpha=p_{0}\left(-\frac{1}{2 \lambda}+\frac{\rho}{2} \frac{\sigma_{z}}{\sigma_{v}}\right)=-p_{0} \beta
$$


where the last equality follows from the expression for $\beta$ in (2.1). Accordingly $\alpha=-\mu \beta=-p_{0} \beta$, and the pricing rule simplifies to $\tilde{p}(x, p)=p_{0}+\lambda y$. Furthermore the insider's demand function can be written $x(\tilde{v})=\beta \tilde{v}+\alpha=$ $\beta\left(\tilde{v}-p_{0}\right)$, which is a very natural result.

It remains to determine $\lambda$ and $\beta$ from the other parameters. Starting with $\beta$ we see that

$$
\beta=\frac{1}{2 \lambda}-\frac{1}{2} \rho \frac{\sigma_{z}}{\sigma_{v}}=\frac{\beta^{2} \sigma_{v}^{2}+2 \beta \rho \sigma_{z} \sigma_{v}+\sigma_{z}^{2}}{2\left(\beta \sigma_{v}^{2}+\rho \sigma_{z} \sigma_{v}\right)}-\frac{1}{2} \rho \frac{\sigma_{z}}{\sigma_{v}},
$$

which means that $\beta$ must satisfy the quadratic equation

$$
\beta^{2}+\rho \frac{\sigma_{z}}{\sigma_{v}} \beta+\frac{\sigma_{z}^{2}}{\sigma_{v}^{2}}\left(\rho^{2}-1\right)=0 .
$$

Only a positive trading intensity is allowed, which means that

$$
\beta=\frac{1}{2} \frac{\sigma_{z}}{\sigma_{v}}\left(\sqrt{4-3 \rho^{2}}-\rho\right)
$$

Finally, using the connection between $\lambda$ and $\beta$ we obtain

$$
\lambda=\frac{\sigma_{v}}{\sigma_{z} \sqrt{4-3 \rho^{2}}} .
$$

This completes the proof of the theorem.

We recall what it means for two random variables to be correlated: In this model there exists a mean zero random variable $\epsilon$ such that $\tilde{z}=a+b \tilde{v}+\tilde{\epsilon}$ with probability one, where $\tilde{\epsilon}$ and $\tilde{v}$ are orthogonal in the meaning $E(\tilde{v} \tilde{\epsilon})=0 .{ }^{1}$ The constant $b$ has the same sign as $\rho$. When $|\rho|=1$ the random variable $\epsilon=0$ with probability one. We may interpret the part $a+b \tilde{v}$ of $\tilde{z}$ as the "informed part" and the term $\tilde{\epsilon}$ as the pure noise trader part. If the insider has less than full information about the true price, his information can be represented in this way, for some non-zero $a$ and $b$. The standard model treats the case $a=b=0$, so that $\tilde{z}=\tilde{\epsilon}$ with probability one.

\section{Properties of the equilibrium}

The first observation we make is that when $\rho=0$, then $\beta=\sigma_{z} / \sigma_{v}$ and $\lambda=\sigma_{v} / 2 \sigma_{z}$ which are the same expressions as given by Kyle (1985). When this is the case, our model is the same as the standard one.

\footnotetext{
${ }^{1}$ In a normal universe this also implies that $\tilde{\epsilon}$ and $\tilde{v}$ are independent.
} 
The results in Theorem 2.1 are so explicit that they enable us to study the effects of the correlation coefficient $\rho$ on the equilibrium.

Starting with the depth of the market, it is equal to $1 / \lambda(\rho)$, and seen to be symmetric in $\rho$ with maximal value $2 \sigma_{z} / \sigma_{v}$ at $\rho=0$, and minimal values $\sigma_{z} / \sigma_{v}$ at $|\rho|=1$ (see Fig. 1 when $\sigma_{z}=\sigma_{v}=1$ ).

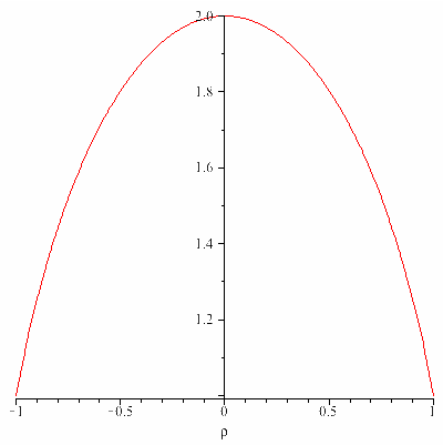

Fig. 1: Market depth $\frac{1}{\lambda(\rho)}$ as a function of $\rho$

This quantity measures the trade, i.e., order flow, necessary to change the price by one unit of account. It is natural that this amount is at its smallest when the information in the market is at its highest, i.e., when the correlation coefficient $\rho$ equals plus or minus one. When $\rho=0$ this measure $1 / \lambda(0)=2 \sigma_{z} / \sigma_{v}$ is proportional to the amount of noise trading to the amount of private information the insider is expected to have. An interpretation is that market makers compensate themselves for the bad trades due to adverse selection of insiders by making the market less liquid. When the noise traders have information $(\rho>0)$, or are manipulated $(\rho<0)$ by the insider, the market makers' need to compensate themselves in this way is diminished.

The liquidity parameter $\lambda(\rho)$ increases as $|\rho|$ increases, which is natural, since the market makers then gradually know more about the true price from the trades made, and thus put more weight on the aggregate market orders when forming prices.

A simple measure of informativeness of prices is defined by

$$
\iota:=1-\frac{\operatorname{var}(\tilde{v} \mid \tilde{p})}{\operatorname{var}(\tilde{v})} .
$$

When the price carries no private information about the true value of the asset, the conditional variance equals the unconditional variance, and $\iota=$ 0 . When the price leaves no uncertainty left in the value of the asset, the 
conditional variance equals zero and $\iota=1$, in which case all the private information is included in the price. Thus $0 \leq \iota \leq 1$.

Using properties of the bivariate normal distribution of $(\tilde{v}, \tilde{p})$, we obtain the expression

$$
\iota(\rho)=\left(1-\frac{\operatorname{var}(\tilde{v} \mid \tilde{p})}{\sigma_{v}^{2}}\right)=\left(1-\frac{\sigma_{z}^{2}\left(1-\rho^{2}\right)}{\beta^{2} \sigma_{v}^{2}+2 \beta \rho \sigma_{z} \sigma_{v}+\sigma_{z}^{2}}\right) .
$$

Inserting the expression for the trading intensity $\beta(\rho)$ of the insider, we obtain

$$
\iota(\rho)=\left(1-\frac{\left(1-\rho^{2}\right)}{2-\frac{3}{2} \rho^{2}+\frac{1}{2} \rho \sqrt{\left(4-3 \rho^{2}\right)}}\right)
$$

in equilibrium. As in the standard model $(\rho=0)$, the measure of informativeness is unaffected by the level of noise trading $\left(\sigma_{z}\right)$. As a function of $\rho$ the measure $\iota(\rho)$ is antisymmetric and increases from 0 when $\rho=-1$ to 1 when $\rho=1 .{ }^{2}$ Figure 2 illustrates.

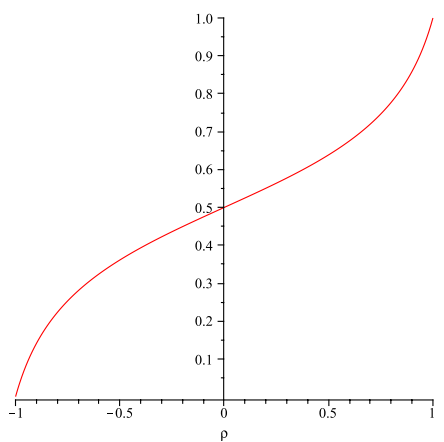

Fig. 2: Informativeness $\iota(\rho)$ as a function of $\rho$

When $\rho=0$ the informativeness $\iota(0)=\frac{1}{2}$, i.e., one-half of the insider's private information is incorporated into prices. When $\rho=1$ all the private information is included in the price, and when $\rho=-1$ the price carries no private information. As $\rho$ increases, trade takes place between more and more equally informed parties, and more information about the true value of the asset becomes revealed in its price. Also the market makers increase the liquidity parameter $\lambda(\rho)$, which works in the same direction. When $\rho$ is negative we interpret this as manipulation, and the informativeness in prices is naturally low.

\footnotetext{
${ }^{2}$ We use L'Hôpital's rule to verify that $\iota(\rho) \downarrow 0$ when $\rho \downarrow-1$.
} 
When $\rho$ increases, information becomes distributed more and more like in a rational expectations equilibrium, although the level of information is much higher.

The trading intensity $\beta(\rho)$ as a function of $\rho$ of Theorem 2.1 is illustrated in Fig. 3 when $\sigma_{z}=\sigma_{v}=1$.

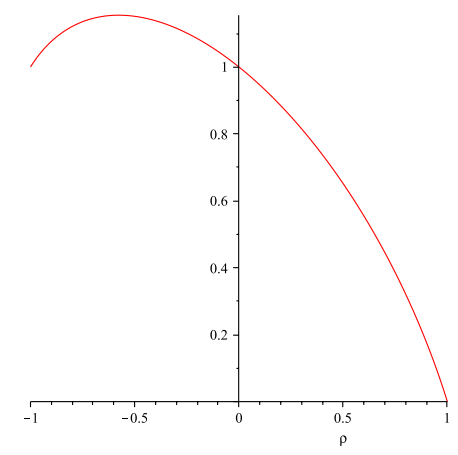

Fig. 3: Trading intensity $\beta(\rho)$ as a function of $\rho$

It has its maximum of $\beta\left(\rho^{*}\right)=\frac{2}{\sqrt{3}} \frac{\sigma_{z}}{\sigma_{v}}$ for $\rho^{*}=-\frac{1}{\sqrt{3}}=-.577$, and decreases to zero as $\rho$ increases to one. The intensity $\beta(\rho)$ increases from $\rho=-1$ to $\rho^{*}$, and it attains the value $\frac{\sigma_{z}}{\sigma_{v}}$ when $\rho$ equals -1 and 0 .

In general will an increase in noise trading bring forth more informed trading, so it does not destabilize prices as long as $\rho<1$. When the correlation becomes perfect, the insider responds by reducing his trade intensity to zero, but since both parties are now in a sense equally well informed, prices are still stable, and the liquidity parameter $\lambda(\rho)$ attains its maximum value of $\frac{\sigma_{v}}{\sigma_{z}}$ as $\rho \rightarrow 1$.

As $\rho$ increases to one, the insider's ability to camouflage his trade is at its minimum. When $\rho$ decreases to $\rho^{*}$, the insider's need to hide his trade decreases, but increases as $\rho$ decreases further from $\rho^{*}$ to -1 .

\subsection{The various profits}

The realized profits of the parties in a one shot auction are the following: The realization of $(\tilde{v}-\tilde{p}) \tilde{x}$ for the noise traders, and similarly the realizations of $(\tilde{v}-\tilde{p}) \tilde{z}$ and $(\tilde{p}-\tilde{v})(\tilde{x}+\tilde{z})$ for the insider and the market makers respectively. The latter follows since the market makers set the price $\tilde{p}$ and faces the final price $\tilde{v}$ at a total quantity $\tilde{y}=\tilde{x}+\tilde{z}$ traded. All these profits may have various signs, but sum to zero with probability one, since we deal with a pure exchange economy. Let us study expected and various conditional expected 
values of these profits. The market makers set prices such that their expected profits is equal to zero. This follows by the rules of conditional expectation:

$$
\begin{gathered}
E\{(\tilde{p}-\tilde{v})(\tilde{x}+\tilde{z})\}=E\{(E(\tilde{v} \mid \tilde{y}) \tilde{y}-\tilde{v} \tilde{y}\}= \\
E\{E(\tilde{v} \tilde{y} \mid \tilde{y})-\tilde{v} \tilde{y}\}=E(\tilde{v} \tilde{y})-E(\tilde{v} \tilde{y})=0,
\end{gathered}
$$

where the third equality follows by iterated expectations. Given that the market makers observe a total quantity traded equal to $y$, the conditional expectation of their profits is

$$
E\{(\tilde{p}-\tilde{v})(\tilde{x}+\tilde{z}) \mid \tilde{y}=y\}=y^{2}\left(\lambda-\frac{\beta \sigma_{v}^{2}+\rho \sigma_{z} \sigma_{v}}{\beta^{2} \sigma_{v}^{2}+\sigma_{z}^{2}+2 \beta \rho \sigma_{z} \sigma_{v}}\right)=0 \quad \text { for all } y
$$

in equilibrium, where we have used the joint normality of the variables involved, and where (2.3) implies the last equality sign. Thus, even if the market makers earn or lose in one single shot of the auction, their conditional expected profit is always zero for any value $y$ of the total quantity traded.

Moving to the insider, also his realized profit in one round of trade may be positive or negative. However, his conditional expected profit given the information he has is the following:

$$
E\{(\tilde{v}-\tilde{p}) \tilde{x} \mid \tilde{v}=v\}=\frac{1}{2}\left(v-p_{0}\right)^{2} \frac{\sigma_{z}}{\sigma_{v}}\left(\frac{2-\rho^{2}-\rho \sqrt{4-3 \rho^{2}}}{\sqrt{4-3 \rho^{2}}}\right) .
$$

From this it follows immediately by averaging this expression over the distribution of $\tilde{v}$ that the expected profits of the insider is given by

$$
E\{(\tilde{v}-\tilde{p}) \tilde{x})=\frac{1}{2} \sigma_{z} \sigma_{v}\left(\frac{2-\rho^{2}-\rho \sqrt{4-3 \rho^{2}}}{\sqrt{4-3 \rho^{2}}}\right) .
$$

Fig. 4 illustrates the latter as a function of $\rho$ when $\sigma_{z}=\sigma_{v}=1$. 


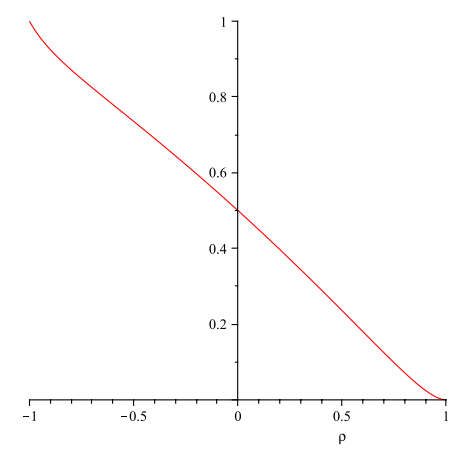

Fig. 4: Insider's average profit as a function of $\rho$

As the figure indicates, the average profit is positive, and a decreasing function of the correlation coefficient $\rho$. When $\rho=-1$ the profit is $\sigma_{z} \sigma_{v}$, when $\rho=0$ it is $\frac{1}{2} \sigma_{z} \sigma_{v}$, and when $\rho=1$ the profit is zero. The insider's ability to mislead the noise traders pays off for him and yields a maximal expected profit when this ability is perfected, which is twice as large as the corresponding profit in the uncorrelated case. When the insider trades against informed noise traders on the other hand, his profit decreases as the noise traders become more informed, and in the limit when $\rho \rightarrow 1$, this profit goes to zero.

This is the situation when we repeat the auction over and over again. The expression for the conditional expected profit given that $\tilde{v}=v$ can be interpreted as what the insider can expect to earn if $v$ is his true inside information about the price of the risky asset. It has the same form as a function of the correlation coefficient $\rho$ as the unconditional expectation, but depends in addition on how far the value $v$ is from the expected value $p_{0}$. The bigger this difference, the more the insider can expect to make. In the gambling analogue, the bigger the difference $\left(v-p_{0}\right)^{2}$, the higher are the odds in favor of the casino.

Finally considering the noise traders, some of them may win and some lose in any single round of the auction and the same can happen in aggregate. However, given that they trade the amount $\tilde{z}=z$, how much can they expect to earn? This amount is

$$
E\{(\tilde{v}-\tilde{p}) \tilde{z} \mid \tilde{z}=z\}=\frac{1}{2} z^{2} \frac{\sigma_{v}}{\sigma_{z}}\left(\frac{\rho^{2}-2+\rho \sqrt{4-3 \rho^{2}}}{\sqrt{4-3 \rho^{2}}}\right) .
$$

In a repeated version of this market, the average profit of the noise traders 
is accordingly

$$
E\{(\tilde{v}-\tilde{p}) \tilde{z}\}=\frac{1}{2} \sigma_{v} \sigma_{z}\left(\frac{\rho^{2}-2+\rho \sqrt{4-3 \rho^{2}}}{\sqrt{4-3 \rho^{2}}}\right) .
$$

Its graph is shown in Fig. 5 as a function of $\rho$ when $\sigma_{z}=\sigma_{v}=1$.

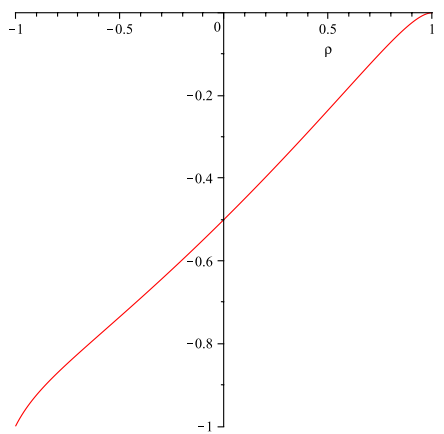

Fig. 5: Noise traders' average profit as a function of $\rho$

By comparing with the insider's profit, it is seen to be exactly the same in absolute value, but with an opposite sign. This is verified by our above results: Since the sum of the profits are zero for all states of the world, the sum of the expected profits must also equal zero. We have shown that the market makers earn zero average profits, so the noise traders profits plus the insiders profits must equal zero on average. This is confirmed by the above calculations.

Returning to our question raised above, given that the noise traders trade the amount $z$, they can merely expect to make negative profits as long as $\rho<1$. This expected loss is larger the larger the amount $z$ traded, which also has a clear counterpart in the gambling analogue mentioned earlier. This conditional expected profit has the same form as the expected profits shown in Fig. 5, as a function of the correlation coefficient $\rho$. When they are equally informed as the insider, i.e., when $\rho=1$, the best they can hope for in expectation (and conditional expectation given $\tilde{z}=z$ ) is zero profits, which is then also the expected profit (and the conditional expected profit given $\tilde{v}=v$ ) of the insider. 


\section{Summary}

The single auction equilibrium of Kyle's (1985) is studied, in which noise traders may be partially informed, or alternatively they can be manipulated. Unlike Kyle's assumption that the quantity traded by the noise traders is independent of the asset value, we assume that the noise traders are able to correlate their trade with the true price.

We found several implications from this ability for the equilibrium, one being that the insider's expected profit decreases as the noise traders' ability to correlate positively improves. This also happens to the insider's conditional expected profits, given his particular inside information. In the limit, the noise traders do not lose on average, and the insider makes zero expected profits. When the correlation is negative, we interpret this as manipulation. In this case the insider makes the highest expected profits, and the informativeness of prices are at its minimum.

The market depth is a symmetric, concave function of the correlation coefficient $\rho$ between the noise traders traded amount and the value of the risky asset. This shows that the market makers' propensity to compensate themselves for bad trades due to adverse selection of insiders is diminished when this correlation $\rho$ moves away from zero.

The informativeness of prices is, on the other hand, an antisymmetric function of the correlation coefficient. This measure is at its minimum of 0 when $\rho=-1$, increases to one half of this when $\rho=0$, and then further increases to 1 when $\rho \rightarrow 1$. This shows that when when speculators become equally well informed, it is easier for the market makers to infer the true price. When insiders mislead the public on the other hand, the informativeness in prices decreases, and at the same time the insider makes his greatest expected profits.

\section{References}

[1] Aase, K, T. Bjuland, and B. Øksendal (2011a). An anticipative linear filtering equation. Systems \& Control Letters, pp 1-4.

[2] Aase, K, T. Bjuland, and B. Øksendal (2011b). Strategic Insider Trading Equilibrium: A Filter Theory Approach (2011). Afrika Matematika (to appear) 
[3] Admati, A. R. and P. Pfleiderer (1988). " A Theory of Intraday Patterns: Volume and Price Variability". The Review of Financial Studies 1, 1, $3-40$.

[4] Back, K. (1992). "Insider Trading in Continuous Time". The Review of Financial Studies Vol. 5, No 3, 387-409.

[5] Barberis, N., and R. Thaler (2003). "A Survey of Behavioral Finance". Handbook of the Economics of Finance: Financial Markets and Asset Pricing, Chapter 18, Vol. 1B (Elsevier North-Holland).

[6] De Long, J. B., A. Shleifer, L. Summers, and R. Waldman (1990). "Noise Traders Risk in Financial Markets." Journal of Political Economy 98, 703-738.

[7] De Marzo, P., and D. Duffie (1999). "A Liquidity-based Model of Security Design". Econometrica 67, 65-99.

[8] Diamond, D., and R. Verrecchia (1981). "Information Aggregation in a Noisy Rational Expectations Economy." Journal of Financial Economics 9. 221-235.

[9] Dow, J., and G. Gorton (2006). "Noise Traders." NBER Working Paper 12256, Cambridge, MA.

[10] Dow, J., and G. Gorton (1994). "Arbitrage Chains". Journal of Finance $49,819-850$.

[11] Glosten, L. R., and P. R. Milgrom (1985). "Bid, Ask and Transaction Prices in a Specialist Market with Hetrogeneously Informed Traders". Journal of Financial Economics, 14, 71-100.

[12] Grossman, S. J., and J. E. Stiglitz (1980). "On the Impossibility of Informationally Efficient Markets". American Economic Review, 70 , 393-408.

[13] Holden, C. W. and A. Subrahmanyam (1992). "Long-Lived Private Information and Imperfect Competition". The Journal of Finance 47, 247270 .

[14] Kyle, A. S. (1985). "Continuous Aucutions and Insider Trading". Econometrica Vol.53, No. 6, 1315-1336. 
[15] Shleifer, A., and L. Summers (1990). "The Noise Trader Approach to Finance". Journal of Economic Perspectives 4, 19-33.

[16] Schleifer, A., and R. Vishny (1997). "The Limits of Arbitrage". The Journal of Finance 52, 35-55.

[17] Shiller, R. J. (1984). "Stock Prices and Social Dynamics". Brookings Papers on Economic Activity, Fall: 457-498.

[18] Spiegel, M., and A. Subrahmanyam (1992). "Informed Speculation and Hedging in a Non-competitive Securities Market". Review of Financial Studies 5, 307-329. 